\title{
A Simple Method for Eccentric Event Espial Using Mahalanobis Metric
}

\author{
Md. Haidar Sharif and Chabane Djeraba \\ University of Sciences and Technologies of Lille (USTL), France \\ \{md-haidar.sharif, chabane.djeraba\}@lifl.fr
}

\begin{abstract}
The paper presents an approach, which detects eccentric events in real time surveillance video systems (e.g., escalators), based on optical flow analysis of multitude behavour followed by Mahalanobis and $\chi^{2}$ metrics. The video frames are flagged as normal or eccentric established on the statistical classification of the distribution of Mahalanobis distances of the normalized spatiotemporal information of optical flow vectors. Those optical flow vectors are computed from the small blocks of the explicit region of successive frames namely Region of Interest Image (RII), which is discovered by RII Map (RIIM). The RIIM is obtained from specific treatment of foreground segmentation of moving subjects. The method essentially has been tested against a single camera data-set.
\end{abstract}

\section{Introduction}

Video surveillance is commonly used in security systems, but requires more intelligent and more robust technical approaches. An automatic video surveillance is attractive because it pledges to replace the more costly option of staffing video surveillance monitors with human observers. There are many applications for systems that can detect emergencies and provide profitable and informative surveillance. For instance, escalators have become an importance portion of metropolitan life. The USCPSC estimates that there are approximately 7300 escalator-related injuries in the United States each year 11. The USCPSC estimated an average of 5900 hospital emergency-room-treated injuries associated with escalators each year between 1990 and 1994. However, large-scale video surveillance of escalators would benefit from a system capable of recognizing eccentric (abnormal) events to make the system operators alert and fully informed. An event is said to be an observable action or change of state in a video stream that would be important for security management. For detecting events, authors in 2 focused on differences in the direction of motion and speed of persons, authors in [3] used optical flow features and support vector machine to detect surveillance events, while authors in 4] heavily relied on the optical flow concept to track feature points for each frame of a video. Optical flow features with Hidden Markov Models were used to detect emergency or eccentric events in the crowd 5[6] but those methods were not experimented on the real world video data-set. We will put forward an approach, which is based on statistical treatments of spatiotemporal (optical flow) patterns of human behaviours, to

E. Bayro-Corrochano and J.-O. Eklundh (Eds.): CIARP 2009, LNCS 5856, pp. 417-424, 2009.

(C) Springer-Verlag Berlin Heidelberg 2009 
detect eccentric events essentially in unidirectional crowd flow (e.g., escalators). We get-go by calculating a Region of Interest Image Map (RIIM) during a period of time to extract the main regions of motion activity. The use of RIIM improves the quality of the results and reduces processing time which is an important factor for real-time applications. The optical flow information, calculated from the Region of Interest Images defined by RIIM in successive frames, of video reflects the crowd multi-modal behaviors as optical flow patterns variate in time. There is sufficient perturbation in the optical flow pattern in the crowd in case of abnormal and/or emergencies situations [see Fig. 1] (c) and (d)]. We calculate Mahalanobis distances using the extracted spatiotemporal information. Mahalanobis metric uses an appropriate correlation matrix to take into account of differences in variable variances and correlations between variables. We study the distribution of Mahalanobis distances along with a defined cutoff value $T_{d}$ to make difference between normal and abnormal frames. To analyze the optical flow patterns of human crowds scenes, we have concentrated on escalator videos to use in our applications. One practical application of our approach is in the detection of real-time collapsing events, which could lead to perilous and inconsistent conditions. The exercised videos are from camera installed at an airport to monitor the situation of mainly escalator exits. The abstraction of the application is to have essentially escalator exits continuously observed to react quickly in the event of any collapsing. With this aim, cameras are installed in front of the exit locations to observe and send the video signal to a control room, where dedicated employees can monitor and respond to the collapsing situations.

The rest of this paper has been organized as follows: Section 2 delineates the proposed framework; Section 3 reports the experimental results; finally, Section 4 presents the conclusion of the work with few inklings for further investigation.

\section{Proposed Approach}

\subsection{Region of Interest Image Map (RIIM)}

The RIIM can be defined automatically by building a color histogram [see Fig. 1] (a) \& (b) for escalator case], which is built from the accumulation of binary blobs of moving subjects, which were extracted following foreground segmentation method [7]. The adaptive background subtraction algorithm proposed by [7] is
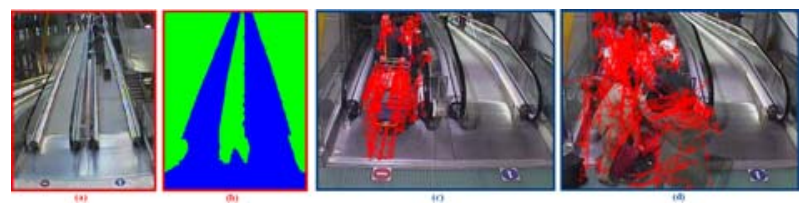

Fig. 1. (a) Camera view. (b) Generated Region of Interest Image Map (RIIM) and blue region on the RIIM recommends Region of Interest Image (RII). Ordered \& disordered optical flow vectors in (c) \& (d) limn normal and abnormal circumstances respectively. 
able to model a background from a long training sequence with limited memory, works well on moving backgrounds, illumination changes, and compressed videos having irregular intensity distributions. The RIIM will be brought into existence mainly off-line. On-line is possible but it makes the system complicated. Off-line is better as the generated RIIM will be more significant and accurate when the video duration will be very long. RIIM improves the quality of the results and reduces processing time which is an imperative factor for real-time applications.

\subsection{Spatiotemporal Information (ST-Info) Extraction}

The Region of Interest Image (RII), ascertained by RIIM, is separated into small blocks. Once we define $n$ (say 1500) points of interest in the RII, we track those points over the small blocks of two successive region of interest images using the combination feature tracker of Kanade-Lucas-Shi-Tomasi [89] easily. To get an acceptable distribution of optical flow pattern over the RII, we take into account vertical coordinate of each block. Consequently, a weighing coefficient $\lambda$ is calculated according to the vertical coordinate of the block. A block far away from the camera has small vertical coordinate, as a result its $\lambda$ should be large. Equally, block with large vertical coordinate get smaller $\lambda$. The value of $\lambda$ heavily depends on the context of application and implementation. For our escalator videos data-set typically $\lambda$ limits $0.6 \leq \lambda \leq 1$. Adjacent to camera (starting of the RII) region the value of $\lambda=0.6$ suits well, whereas $\lambda$ bears the maximum value 1 at the opposite end. We also take down the static and noise features. Static features are the features which moves less than two pixels. Noise features are the isolated features which have a big angle and distance difference with their near neighbors due to tracking calculation errors. Finally, for each frame [such as Fig. 1 (c) \& (d)] irrespective of normal or eccentric events, we obtain an acceptable and workable spatiotemporal information, i.e., 5 features are observed in time and put in the form of a $n \times 5$ matrix $\mathbf{M}(\mathbf{j})(\mathbf{k})$ by dint of:

$$
\mathbf{M}(\mathbf{j})(\mathbf{k})=\left[\begin{array}{lllll}
x(1)(1) & x(2)(1) & x(3)(1) & x(4)(1) & x(5)(1) \\
x(\dot{1})(i) & x(2)(i) & x(3)(i) & x(4)(i) & x(5)(i) \\
x(\dot{1})(n) & x(2)(n) & x(3)(n) & x(4)(n) & x(5)(n)
\end{array}\right]
$$

where $j=1,2,3,4,5 ; k=1,2, \ldots, n ; i$ be a feature element in $k$; and $x(1)(i) \mapsto$ $x$-coordinate of the $i, x(2)(i) \mapsto y$-coordinate of the $i, x(3)(i) \mapsto x$-velocity with multiply by a weighing coefficient $\lambda_{i}$ of the $i, x(4)(i) \mapsto y$-velocity with multiply by a weighing coefficient $\lambda_{i}$ of the $i, x(5)(i) \mapsto$ acting motion direction of the $i$.

\subsection{Statistical Treatments of the ST-Info}

Normalization of Raw Data: A normalized value is a value that has been processed in a way that makes it possible to be efficiently compared against other values. For each column of $\mathbf{M}(\mathbf{j})(\mathbf{k})$, we calculate the average $\overline{x_{j}}$ and standard deviation $\sigma_{j}$. Subtracting the average $\overline{x_{j}}$ from each value in the columns of $x(j)(k)$, and then dividing by the standard deviation $\sigma_{j}$ for that column in 
$x(j)(k)$ generated a new matrix $z(j)(k)$ as: $\quad \overline{x_{j}}=\frac{1}{n} \sum_{k=1}^{n} x(j)(k), \quad \sigma_{j}=$ $\sqrt{\frac{\sum\left(x(j)(k)-\overline{x_{j}}\right)^{2}}{n-1}}, \quad z(j)(k)=\frac{x(j)(k)-\overline{x_{j}}}{\sigma_{j}}$. All values in $z(j)(k)$ are dimensionless and normalized, consequently, the new pattern of the $\mathbf{M}(\mathbf{j})(\mathbf{k})$ gives right of way:

$$
\mathbf{Z}(\mathbf{j})(\mathbf{k})=\left[\begin{array}{lllll}
z(1)(1) & z(2)(1) & z(3)(1) & z(4)(1) & z(5)(1) \\
z(\dot{1})(i) & z(2)(i) & z(3)(i) & z(4)(i) & z(5)(i) \\
z(1 \dot{)}(n) & z(2 \dot{)}(n) & z(3 \dot{)}(n) & z(4)(n) & z(5)(n)
\end{array}\right] .
$$

A covariance matrix is merely collection of several variance-covariances in the form of a square matrix. But one problem with covariance is that it is sensitive to the scales. To obtain a more direct indication of how two components covary, we scale covariance to obtain correlation. Correlation is dimensionless while covariation is in units obtained by multiplying the units of each variable. Using $\mathbf{Z}(\mathbf{j})(\mathbf{k})$, scaling is performed by means of the following equations: $\quad r_{p q}=\frac{S_{p q}}{S_{p} S_{q}}$, $S_{p q}=\frac{1}{n-1} \sum_{k=1}^{n}\left[z_{p}(k) z_{q}(k)\right], S_{l}=\sqrt{\frac{1}{n-1} \sum_{k=1}^{n}\left[z_{l}(k)^{2}\right]}, \quad\{p, q\} \in j, \quad l \in\{p, q\}$.

Calculation of Mahalanobis Distance $D_{m}(i)$ : In statistics, Mahalanobis distance is based on correlations between variables by which different patterns can be identified and analyzed. It is a useful way of determining similarity of an unknown sample set to a known one. It differs from Euclidean distance in that it takes into account the correlations of the data set and is scale-invariant, i.e., not dependent on the scale of measurements. The region of constant Mahalanobis distance around the mean forms an ellipse in two dimensional space (i.e., when only 2 variables are measured), or an ellipsoid or hyperellipsoid when more variables are used. The Mahalanobis distance is the same as the Euclidean distance if the correlation matrix is the identity matrix. We calculate the Mahalanobis distance $D_{m}(i)$ for each row of the normalized matrix $\mathbf{Z}(\mathbf{j})(\mathbf{k})$ by multiplying the row by the inverted correlation matrix, then multiplying the resulting vector by the transpose of the row of the $\mathbf{Z}(\mathbf{j})(\mathbf{k})$, then dividing the obtained result by the degree of freedom, finally grasping square root of the up-to-the-minute result as:

$$
D_{m}(i)=\sqrt{\left[\frac{z(1)(i) z(2)(i) z(3)(i) z(4)(i) z(5)(i)}{5}\right]\left[\begin{array}{ccccc}
1 & r_{12} & r_{13} & r_{14} & r_{15} \\
r_{21} & 1 & r_{23} & r_{24} & r_{25} \\
r_{31} & r_{32} & 1 & r_{34} & r_{35} \\
r_{41} & r_{42} & r_{43} & 1 & r_{45} \\
r_{51} & r_{52} & r_{53} & r_{54} & 1
\end{array}\right]^{-1}\left[\begin{array}{l}
z(1)(i) \\
z(2)(i) \\
z(3)(i) \\
z(4)(i) \\
z(5)(i)
\end{array}\right]}
$$

where the number of columns contained in $\mathbf{Z}(\mathbf{j})(\mathbf{k})$ is referred to as the degree of freedom which is 5 in this case. Geometrically, samples with an equal $D_{m}(i)$ lie on an ellipsoid (Mahalanobis Space). The $D_{m}(i)$ is small for samples lying on or close to the principal axis of the ellipsoid. Samples further away from the principal axis have a much higher $D_{m}(i)$. The larger the $D_{m}(i)$ for a sample is, the more likely the sample is an outlier. An outlier (extreme sample) is a sample that is very different from the average sample in the data set. An outlier may be an ordinary sample, but of which at least one attribute has been severely corrupted by a mistake or error (e.g., tracking calculation errors). An outlier 
may also be a bona fide sample, that simply turns out to be exceptional. Since Mahalanobis distance satisfies the conditions (symmetry, positivity, triangle inequality) of metric, it is a metric. The use of the Mahalanobis metric removes several limitations of the Euclidean metric: (i) it automatically accounts for the scaling of the coordinate axes, (ii) it corrects for correlation between the different features, (iii) it can provide curved as well as linear decision boundaries. But, there is a disbursement to be paid for those advantages. The computation of the correlation matrix can give rise to problems. When the investigated data are measured over a large number of variables, they can keep under control much redundant or correlated information. This is so-called multicollinearity in the data which leads to a singular correlation matrix that cannot be inverted. Another precinct for the calculation of the correlation matrix is that the number of samples in the data set has to be larger than the number of variables. Yet, in the proposed approach, both problems have been minimized by dint of 5 variables and tracking about 1500 samples (points of interest) in each frame respectively.

\subsection{Classification of Mahalanobis Distances and $T_{d}$ Estimation}

Mahalanobis squared distances are calculated in units of standard deviation from the group mean. Therefore, the calculated circumscribing ellipse formed around the samples actually defines the one standard deviation of that group. This allows the designing of a statistical probability to that measurement. In theory, Mahalanobis squared distance is distributed as a $\chi^{2}$ statistic with degree of freedom equal to the number of independent variables in the analysis. The $\chi^{2}$ distribution has only one parameter called the degree of freedom. The shape of a $\chi^{2}$ distribution curve is skewed for very small degrees of freedom and it changes drastically as the degrees of freedom increase. Eventually, for large degrees of freedom, the $\chi^{2}$ distribution curve looks like a normal distribution curve. Like all other continuous distribution curves, the total area under a $\chi^{2}$ distribution curve is 1.0. The three sigma rule, or 68-95-99.7 rule, or empirical rule, states that for a normal distribution, about $68 \%, 95 \%, 99.7 \%$ of the values lie within 1 , 2 , and 3 standard deviation of the mean respectively. Clearly, almost all values lie within 3 standard deviations of the mean. Consequently, samples that have a squared Mahalanobis distance larger than 3 have a probability less than 0.01 . These samples can be classified as members of non-member group. Samples those have squared Mahalanobis distances less than 3 are then classified as members of member group. The determination of the threshold depends on the application and the type of samples. In the proposed approach, we settle each $D_{m}(i)$ goes either member group or non-member group. Sample with a higher $D_{m}(i)$ than $\sqrt{3}$ is treated as non-member group, otherwise member group. Member group contains absolutely the samples of a normal event, whereas non-member group contains essentially samples of eccentric events (including outliers). Figure 2 depicts, while Mahalanobis metric produces elliptical cluster where samples are well correlated, Euclidean metric produces circular subsets. The non-member group consists of samples $S_{1}, S_{2}, S_{3}, S_{4}, S_{5}, S_{6}, S_{7}$, and the outlier $S_{8}$, while the member group groups the rest samples. Presuming in any non-member group, having $M$ 


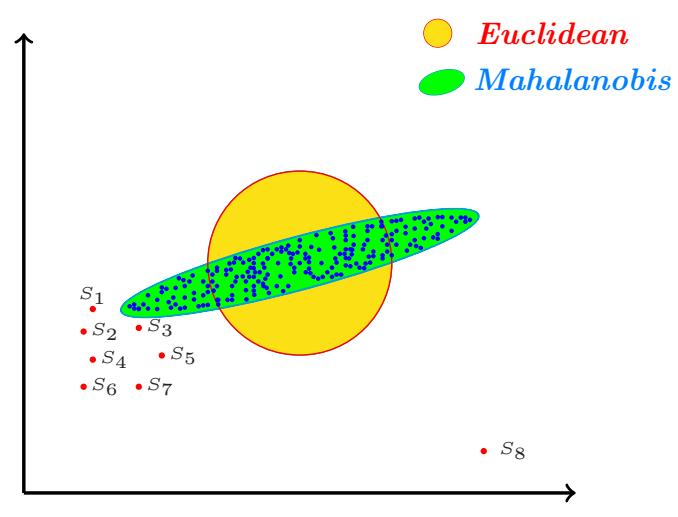

Fig. 2. Mahalanobis metric with respect to Euclidean metric. Samples $S_{1}, S_{2}, S_{3}, S_{4}, S_{5}$, $S_{6}, S_{7}$, and outlier $S_{8}$ go to non-member group, while the rests are member group.

samples including outliers (where also assuming that in general $M \gg$ outliers satisfies), we sum up their Mahalanobis distances as: $S_{d}=\sum_{i=1}^{M} D_{m}(i)$. Now, we transfer each $S_{d}$ into a normalized distance (probability) value ranges between 0 and 1 . The normalization may be done by using the simple formula like $1 / \log \left(S_{d}\right)$, but the normalized values fall into a congested range (scaling problem) which will arise problem specially in threshold selection. To solve the scaling problem, we take the advantage of cumulative distribution function $(c d f)$, which has strict lower and upper bounds between 0 and 1, we can easily pick up the normalized distance of each $S_{d}$. Since all values of $S_{d}$ are skewed to the right (positive-definite) and their variances are also large, we can use Log-normal distribution. Skewed distributions are particularly common when mean values are low, variances large, and values cannot be negative. Log-normal distributions are usually characterized in terms of the log-transformed variable, using as parameters the expected value, or mean (location parameter $\mu$ ), of its distribution, and the standard deviation (scale parameter $\sigma$ ). The $\sigma$ is entitled as scale as its value determines the scale or statistical dispersion of the probability distribution. If $N_{d}$ be the normalized value of $S_{d}$, then $N_{d}$ can be gently estimated by means of:

$$
N_{d}=\frac{1}{2}\left[1+\operatorname{erf}\left\{\frac{\log \left(S_{d}\right)-\mu}{\sigma \sqrt{2}}\right\}\right], \operatorname{erf}(r)=\frac{2}{\sqrt{\pi}}\left[r-\frac{r^{3}}{3}+\frac{r^{5}}{10}-\frac{r^{7}}{42}+\ldots\right]
$$

where erf is a Gauss error function and $r=\frac{\log \left(S_{d}\right)-\mu}{\sigma \sqrt{2}}$. Using Eq. 4, and placing congenial values of $\mu$ and $\sigma$ (say $\mu=0, \sigma=5$ ) we can explicitly estimate the value of $N_{d}$ between 0 and 1 . Now, it is important to define an appropriate threshold $T_{d}$ to make a distinction between normal and abnormal frames. We make a similitude measure between $N_{d}$ and $T_{d}$ to reach an explicit conclusion for each frame, i.e., a frame is said to be eccentric if $N_{d}>T_{d}$, otherwise normal. We estimate $T_{d}$ from video stream which contains none but normal motions using:

$$
T_{d}=\sqrt{\left[\arg \max _{i=1 \ldots f}\left[N_{d}\right]_{i}\right]^{2}+\left[\arg \min _{i=1 \ldots f}\left[\frac{2}{\pi^{2}} \sum_{n=0}^{\infty} \frac{(-1)^{n}\left(N_{d}\right)^{2 n+1}}{n !(2 n+1)}\right]_{i}\right]^{2}}
$$


where $f$ be the total number of frames. The $T_{d}$ extremely depends on the controlled environment. If the video stream changes, then $T_{d}$ should be regenerated.

\section{$3 \quad$ Experimental Results}

To conduct experiments we used 16 real videos, taken in spanning days and seasons, of frame size $640 \times 480$ pixels, collected by cameras installed in an airport to monitor especially the escalator egresses, provided by a video surveillance company (escalator video frames in Fig. 3 have been cropped/grayed for confidential reasons). The videos were used to provide informative data for the security team who may need to take prompt actions in the event of a critical situation such as collapsing. Each video stream consists of normal and eccentric events. The normal situations correspond to crowd flows without any eccentric event on the escalator elsewhere. Eccentric events correspond to videos which contain collapsing events mostly in the escalator egresses. Generally, in the videos we have two escalators corresponding to two-way-traffic of opposite directions. The 1st image (from left) of Fig. 3 describes a scenario of a collapsing event in an escalator exit point. Some stuffs from a heavily loaded trolley have dropped just the egress point of the moving escalator which has caused one kind of emergency situation on the egress point. The 2nd image figures another example of an abnormal event where a wheel from the trolley has suddenly been broken off by the friction during its travel over an escalator and finally on the escalator exit point one kind of perilous and inconsistent circumstances has been come off. The situations have been detected by the proposed algorithm. But the algorithm does not work two of the video streams where video frames bear the situations like Fig. 3 (a) and (b) as the video sequences which include abnormal events have occurred with occlusion. Thus the quantity of extracted optical flow vectors is not sufficient to draw out abnormal frames. Of course, occlusion handling is a difficult part of optical flow technique. Occluded pixels violate a major assumption of optical
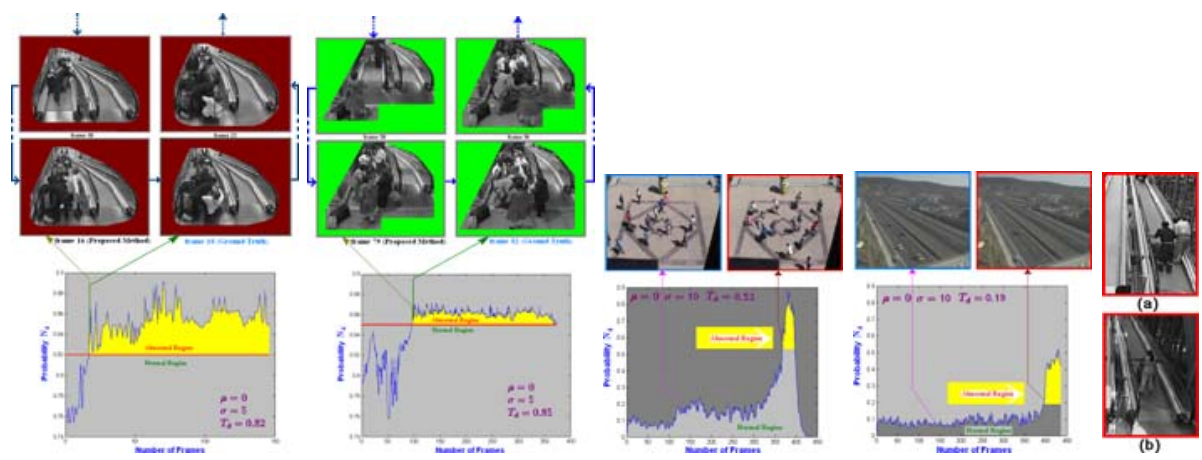

Fig. 3. Curves are the outputs of algorithm. Eccentric events on escalator exits (from left 1st two images), a sudden run of mob (3rd), turnabout of car on high-way (4th) have been detected. But occluded abnormal events in (a) and (b) can not be detected. 
flow technique that each pixel goes somewhere. However, the detection results have been compared with ground truth. Ground truth is the process of manually marking what an algorithm is expected to output. Beyond the escalator unidirectional flow of mob videos, the method has been tested on the videos existing both normal and abnormal events, attributed $320 \times 240$ pixels, where the movements of people are random directions or where cars violate the traffic rules on the high-way, e.g., scenarios depicted on the 3rd and 4th images of Fig. 3. In the 3rd image, people has tended to leave their places with very quick motion. The 4th image concerns the scenario of breaking high-way traffic rules. When the car has tried to make a turnabout, it has broken the traffic rules which has been detected by the algorithm. The blue colored curves are the output of the algorithm. The yellow colored regions represent the abnormal motion activities.

\section{Conclusion}

We evinced a method which detects abnormal events in real time surveillance video systems. ST-Info has been extracted from the small blocks of the RII discovered by RIIM, which improves the quality of results and reduces processing time. The study of the distribution of Mahalanobis distances with predefined threshold $T_{d}$ provides the knowledge of the state of abnormality. Efficacy of the algorithm has been evaluated on the real world crowd scenes. Obtained results, have been compared with ground truths, show the effectiveness of the method on detecting abnormalities. Yet, future work will make suit the method for the cases e.g., a normal event makes less visible or unclear an abnormal event, etc.

\section{References}

1. Commission, U.C.P.S.: Cpsc document \#5111: Escalator safety. The United States Consumer Product Safety Commission (USCPSC), Washington, DC (2003)

2. Kawai, Y., Takahashi, M., Sano, M., Fujii, M.: High-level feature extraction and surveillance event detection. In: NHK STRL at TRECVID (2008)

3. Hao, S., Yoshizawa, Y., Yamasaki, K., Shinoda, K., Furui, S.: Tokyo Tech at TRECVID (2008)

4. Orhan, O.B., Hochreiter, J., Poock, J., Chen, Q., Chabra, A., Shah, M.: Content based copy detection and surveillance event detection. In: UCF at TRECVID (2008)

5. Andrade, E.L., Blunsden, S., Fisher, R.B.: Hidden markov models for optical flow analysis in crowds. In: ICPR 2006, pp. 460-463 (2006)

6. Andrade, E.L., Blunsden, S., Fisher, R.B.: Modelling crowd scenes for event detection. In: ICPR 2006, pp. 175-178 (2006)

7. Kim, K., Chalidabhongse, T.H., Harwood, D., Davis, L.: Real-time foregroundbackground segmentation using codebook model. Real-Time Imaging 11, 172-185 (2005)

8. Lucas, B.D., Kanade, T.: An iterative image registration technique with an application to stereo vision. In: IJCAI 1981, pp. 674-679 (1981)

9. Shi, J., Tomasi, C.: Good features to track. In: CVPR 1994, pp. 593-600 (1994) 